\title{
Magnetic and electric control of spin- and valley-polarized transport across tunnel junctions on monolayer $\mathrm{WSe}_{2}$
}

\author{
M. Tahir, ${ }^{1,{ }^{*}}$ P. M. Krstajić,,${ }^{2, \dagger}$ and P. Vasilopoulos ${ }^{3, \ddagger}$ \\ ${ }^{1}$ Department of Physics, College of Science, University of Hafr Al Batin, P.O. Box 1803, Hafr Al Batin 31991, Kingdom of Saudi Arabia \\ ${ }^{2}$ Center of Microelectronic Technologies and Single Crystals (IHTM), University of Belgrade, Njegoševa 12, 11000 Belgrade, Serbia \\ ${ }^{3}$ Department of Physics, Concordia University, 7141 Sherbrooke West, Montreal, Quebec, Canada H4B 1R6
}

(Received 25 March 2017; published 1 June 2017)

\begin{abstract}
The recent experimental realization of high-quality $\mathrm{WSe}_{2}$ leads to the possibility of an efficient manipulation of its spin and valley degrees of freedom. Its electronic properties comprise a huge spin-orbit coupling, a direct band gap, and a strong anisotropic lifting of the degeneracy of the valley degree of freedom in a magnetic field. We evaluate its band structure and study ballistic electron transport through single and double junctions (or barriers) on monolayer $\mathrm{WSe}_{2}$ in the presence of $\operatorname{spin} M_{s}$ and valley $M_{v}$ Zeeman fields and of an electric potential $U$. The conductance versus the field $M_{s}$ or $M_{v}$ decreases in a fluctuating manner. For a single junction, the spin $P_{s}$ and valley $P_{v}$ polarizations rise with $M=M_{v}=2 M_{s}$, reach a value of more than 55\%, and become perfect above $U \approx 45 \mathrm{meV}$ while for a double junction this change can occur for $U \geqslant 50 \mathrm{meV}$ and $M \geqslant 5 \mathrm{meV}$. In certain regions of the $(M, U)$ plane $P_{v}$ becomes perfect. The conductance $g_{c}$, its spin-up and spin-down components, and both polarizations oscillate with the barrier width $d$. The ability to isolate various carrier degrees of freedom in $\mathrm{WSe}_{2}$ may render it a promising candidate for new spintronic and valleytronic devices.
\end{abstract}

DOI: 10.1103/PhysRevB.95.235402

\section{INTRODUCTION}

Currently, there is a strong interest in two-dimensional (2D) systems as potential hosting materials for applications in spintronics and valleytronics [1]. Similar to graphene [2-4], the first hexagonal Brillouin zone of group-VI monolayer transition metal dichalcogenides (TMDCs) (e.g., $M X_{2}, M=\mathrm{Mo}$ and $\mathrm{W} ; X=\mathrm{S}, \mathrm{Se}$, and Te) [5-10] accommodates pairs of inequivalent valleys. Distinctively, the monolayer $M X_{2}$ exhibits a huge gap and strong spin-orbit coupling (SOC). This enables valley-dependent optical selection rules which allow for valley polarization and spin-valley coupling. The valence and conduction band extrema are located at both $K$ and $K^{\prime}$ valleys at the corners of the hexagonal Brillouin zone. The degenerate $K$ and $K^{\prime}$ valleys are related to each other by time-reversal symmetry and give rise to the valley degree of freedom of the band-edge electrons and holes [11-14]. It has been demonstrated that monolayer $\mathrm{MoS}_{2}$ has reasonable inplane carrier mobility, high thermal stability, and good compatibility with standard semiconductor manufacturing [15]. These properties render monolayer $\mathrm{MoS}_{2}$ a promising candidate for a wide range of applications, including photoluminescence at visible wavelengths $[11,16]$, photodetectors with high responsivity [13], and field-effect transistors [15,17,18].

In contrast to the extensive theoretical [19] and experimental efforts on spin- and valley-controlled applications of $\mathrm{MoS}_{2}$ as well as of silicine [20-22], the high quality of $\mathrm{WSe}_{2}$ [23] and its much stronger SOC, $2 \lambda_{v}^{\prime}=450 \mathrm{meV}$ in the valence band and $2 \lambda_{c}^{\prime}=30 \mathrm{meV}$ in the conduction band, provide an excellent system for spin- and valley-controlled materials $[24,25]$. Although monolayer $\mathrm{WSe}_{2}$ is a direct bandgap semiconductor $(2 \Delta=1.7 \mathrm{eV})$, the lifting of the valley

\footnotetext{
*m.tahir06@alumni.imperial.ac.uk

†predrag222@gmail.com

p.vasilopoulos@concordia.ca
}

degeneracy allows for optical manipulation of the electron valley index or degree of freedom, e.g., by an external magnetic field applied perpendicular to the 2D layer [24,25]. The lifting of the degeneracy of the two valleys in $\mathrm{WSe}_{2}$ monolayers is achieved by monitoring the energy splitting between the two circularly polarized luminescence components, $\sigma^{+}$and $\sigma^{-}$, associated with optical recombination in the nonequivalent valleys. These studies mark only the beginning of investigation of optical transitions in monolayer $\mathrm{WSe}_{2}$ and related compounds in magnetic fields. Direct optical transitions in monolayer $\mathrm{WSe}_{2}$ occur at the edge of the Brillouin zone, which mainly consists of strongly localized $d$ orbitals of the transition metal. This is in contrast with GaAs and other conventional semiconductors used in optoelectronics, where the direct optical band gap is situated at the center of the Brillouin zone. In monolayer $\mathrm{WSe}_{2}$ there are several possible contributions to the Zeeman splitting as the emission of circularly polarized light originates from states with contrasting valley index, spin, and orbital magnetic moment. As the valleys can be selectively addressed, these experiments can determine the different contributions to the Zeeman splitting. Moreover, recently, $\mathrm{WSe}_{2}$ transistors have been demonstrated with high mobility at room temperature [26]. In addition, the advantage of $\mathrm{WSe}_{2}$ over, for example, silicine is that we do not need large perpendicular electric fields [22] to achieve a significant gap.

As the major goal of spintronics and valleytronics, the electrical manipulation of spin and valley injection and transport is essential to realize practical spintronics and valleytronics applications. In light of the above discussion, in this contribution we theoretically propose a single or double biased magnetic barrier in monolayer $\mathrm{WSe}_{2}$ to tune charge transport through it. We find that fully valley-polarized currents, and partially spin-polarized currents, can be obtained simultaneously in this device. We emphasize that the spin splitting near the valence and band edges is essential to the valley-polarized transport, and this characteristic exists in $\mathrm{WSe}_{2}$. Importantly, both the amplitude and direction of the spin-polarized currents can be 
conveniently modulated by the spin and valley Zeeman terms as well as by the bias applied to the barriers without applying a magnetic field. This is in contrast with (gapless) graphene in which a strong valley polarization has been reported in the presence of a magnetic field [27]. The conditions for realizing the fully valley- and spin-polarized conductance are obtained analytically. These results show that TMDCs are very promising materials for spintronics and valleytronics and open up an avenue for electric and or magnetic control of valley and spin polarized transport in $\mathrm{WSe}_{2}$. At zero bias, we show that it is possible to achieve spin $P_{s}$ and valley $P_{v}$ polarization close to $55 \%$ for reasonable values of $M_{s}$ and $M_{v}$ using a single barrier. Applying a bias on the middle layer one can achieve perfect valley polarization $P_{v}$ for $M \geqslant 15 \mathrm{meV}$ and $U \geqslant 90 \mathrm{meV}$ ). For a double barrier the dependence of the conductivity is similar to that of a single barrier but with lower values. Again $P_{v}$ can achieve large values (close to 100\%) in certain regions of the $(M, U)$ plane but $P_{s}$ is in general smaller.

The paper is organized as follows. In Sec. II, we present the model of a junction on $\mathrm{WSe}_{2}$ and basic expressions for the conductance and polarization. In Sec. III, we present and discuss various numerical results, and in Sec. IV, a summary.

\section{MODEL AND BASIC EXPRESSIONS}

We consider a monolayer of $\mathrm{WSe}_{2}$ in the $(x, y)$ plane in the presence of intrinsic SOC, spin, and valley Zeeman fields. The 2D Dirac-like Hamiltonian [23] of $\mathrm{WSe}_{2}$ is

$$
\begin{aligned}
H_{s_{z}}^{\eta}= & v\left(\eta \sigma_{x} p_{x}+\sigma_{y} p_{y}\right)+\Delta \sigma_{z}+\eta s_{z}\left(\lambda_{c} \sigma_{+}+\lambda_{v} \sigma_{-}\right) \\
& +s_{z} \mathrm{M}_{s}-\eta \mathrm{M}_{v}+U(x)
\end{aligned}
$$

Here, $\eta= \pm 1$ for valleys $K$ and $K^{\prime}, \Delta$ is the mass term that breaks the inversion symmetry, $\lambda_{c}=\lambda_{c}^{\prime} / 2$ and $\lambda_{v}=\lambda_{v}^{\prime} / 2$. Furthermore, $\left(\sigma_{x}, \sigma_{y}, \sigma_{z}\right)$ are the Pauli matrices for the valence and conduction bands, and $\sigma_{ \pm}=\sigma_{0} \pm \sigma_{z} ; v\left(5 \times 10^{5} \mathrm{~m} / \mathrm{s}\right)$ denotes the Fermi velocity of Dirac fermions. Spins up and down are denoted by $s_{z}=+1$ and -1 , respectively. Further, $M_{s}$ is the Zeeman field induced by magnetic order, while $M_{v}$ breaks the valley symmetry of the levels. In principle, they can be induced by an external magnetic field, $M_{S}=$ $g^{\prime} \mu_{B} B / 2, M_{s}=g_{v} \mu_{B} B / 2$, where $g^{\prime}$ the Landé $g$ factor $\left(g^{\prime}=\right.$ $\left.g_{e}^{\prime}+g_{s}^{\prime}\right)$, and $\mu_{B}$ the Bohr magneton [24,25]. Also, $g_{e}^{\prime}=2$ is the free electron $g$ factor and $g_{s}^{\prime}=0.21$ is the out-of-plane factor due to the strong SOC in $\mathrm{WSe}_{2}$. The last term in Eq. (1) is the electric potential of the magnetic barrier. For a single barrier, it is given by $U(x)=U\left[\theta\left(x-x_{0}\right)-\theta\left(x-x_{0}-d\right)\right]$, where $\theta(x)$ is the Heaviside function and $d$ the barrier width ( $x_{0}$ marks the beginning of the barrier).

Finally, for the valley degree freedom one has $g_{v}^{\prime}=4$ [24,25]. In addition, an enhanced valley splitting can be achieved by an interfacial magnetic Zeeman field (MEF) produced by placing a monolayer $\mathrm{WSe}_{2}$ on a substrate made of magnetic EuS [28]. First-principle calculations have shown that $\mathrm{EuO}$ can induce a considerable exchange interaction, or Zeeman field effects, in monolayer $\mathrm{MoTe}_{2}$ on top of it, between the Mo and interface Eu atoms, which have unpaired $4 d$ ( $d$ orbitals) electrons, see Refs. [10,28]. We expect similar findings in, e.g., monolayer $\mathrm{WSe}_{2}$ on top of $\mathrm{EuO}(111)$. For $\mathrm{WSe}_{2}$ on top of EuO the magnetic moment of the $f$ electrons
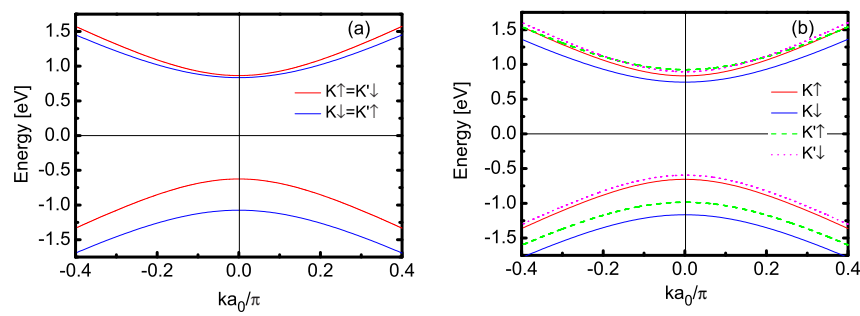

FIG. 1. Dispersion $E(k)$ in $\mathrm{WSe}_{2}$ for (a) $M_{s}=M_{v}=0$ and (b) $M_{s}=30 \mathrm{meV}$ and $M_{v}=60 \mathrm{meV}$. In both panels, $\Delta=0.85 \mathrm{eV}$, $\lambda_{v}=112.5 \mathrm{meV}$, and $\lambda_{c}=7.5 \mathrm{meV}$.

in Eu leads to an exchange interaction between them and the $d$ ones of $\mathrm{W}$ by the magnetic proximity effect. This spin polarizes the $d$ electrons in $\mathrm{W}$ which in turn induce a valley-dependent spin splitting.

By diagonalizing the Hamiltonian given in Eq. (1), we obtain the eigenvalues

$$
E_{s_{z}, t}^{\eta}=\eta s_{z}\left(\lambda_{c}+\lambda_{v}\right)+s_{z} \mathrm{M}_{s}-\eta \mathrm{M}_{v}+U(x)+t E_{s_{z}}^{\eta},
$$

with $E_{s_{z}}^{\eta}=\left[(\hbar v k)^{2}+\left[\Delta+\eta s_{z}\left(\lambda_{c}-\lambda_{v}\right)\right]^{2}\right]^{1 / 2}$. The corresponding eigenfunctions are

$$
\Psi_{s_{z}, t}^{\eta}=\left(e^{i \mathbf{k} \cdot \mathbf{r}} / D_{k}\right)\left(\begin{array}{c}
\eta c_{k} e^{-\eta i \theta} \\
b_{k}
\end{array}\right),
$$

where $c_{k}=\hbar v k, \quad b_{k}=-\Delta-\eta s_{z}\left(\lambda_{c}-\lambda_{v}\right)+t E_{s_{z}}^{\eta}, \quad D_{k}=$ $\left[c_{k}^{2}+\left[\Delta+\eta s_{z}\left(\lambda_{c}-\lambda_{v}\right)-t E_{s_{z}}^{\eta}\right]^{2}\right]^{1 / 2}$, and $\theta=\tan ^{-1}\left(k_{y} / k_{x}\right)$. Further, $t=1(-1)$ denotes the conduction (valence) band and $k$ is the $2 \mathrm{D}$ wave vector.

The spectrum (2) is shown in Fig. 1 versus $k a_{0}$ (where $a_{0}=0.331 \mathrm{~nm}$ is the lattice constant) for two cases: (a) $\mathrm{M}_{s}=$ $\mathrm{M}_{v}=0$ and (b) $M_{s}=30 \mathrm{meV}$ and $M_{v}=60 \mathrm{meV}$. As can be seen, the valence band has a larger splitting even when the Zeeman field is present. We find that there is a valley asymmetry due to the application of magnetic field. This can be understood from the valley Zeeman term $M_{v}$ in Eq. (2), which makes the two valleys nondegenerate in both bands. This clearly confirms the magnetic field-controlled valley pseudospin degree of freedom in $\mathrm{WSe}_{2}$.

We now use these considerations to study ballistic electron transport across a magnetic junction in $\mathrm{WSe}_{2}$ with a metallic gate above it which extends over a region of width $d$ (see Fig. 2). We assume that regions I and III in Fig. 2 are made of the usual $\mathrm{WSe}_{2}$ [no Zeeman, $M_{s}=M_{v}=0$ and

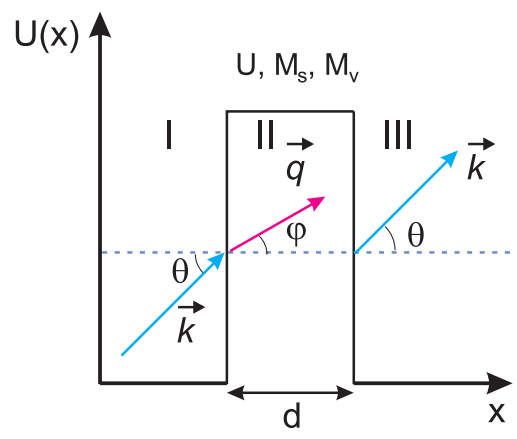

FIG. 2. Schematics of a single junction or barrier on $\mathrm{WSe}_{2} . M_{s}$ and $M_{v}$ are Zeeman fields for spin and valley degrees of freedom, while $U(x)$ is the potential energy. 
no potential barrier $U(x)=0$ ], while a magnetic barrier is placed in region II $(U(x) \neq 0)$ in which the full Hamiltonian (1) applies. For $x<0$, the wave functions are

$$
\begin{aligned}
& \Psi_{1}(x, y) \\
& \quad=e^{i \mathbf{k} \cdot \mathbf{r}}\left(\begin{array}{c}
A_{1} e^{-i \eta \theta} \\
B_{1}
\end{array}\right)+r_{\eta s_{z}} e^{i\left(-k_{x} x+k_{y} y\right)}\left(\begin{array}{c}
A_{1} e^{-i \eta(\pi-\theta)} \\
B_{1}
\end{array}\right),
\end{aligned}
$$

where $A_{1}=\eta c_{k} / D_{k}$ and $B_{1}=b_{k} / D_{k}$. The result

$$
\begin{aligned}
\Psi_{2}(x, y)= & P e^{i\left(q_{x} x+k_{y} y\right)}\left(\begin{array}{c}
A_{2} e^{-i \eta \varphi} \\
B_{2}
\end{array}\right) \\
& +Q e^{i\left(-q_{x} x+k_{y} y\right)}\left(\begin{array}{c}
A_{2} e^{-i \eta(\pi-\varphi)} \\
B_{2}
\end{array}\right) .
\end{aligned}
$$

is for $0<x<d$. Finally, for $x>d$, we have

$$
\Psi_{3}(x, y)=t_{\eta s_{z}} e^{i \mathbf{k} \cdot \mathbf{r}}\left(\begin{array}{c}
A_{1} e^{-i \eta \theta} \\
B_{1}
\end{array}\right) .
$$

Here, $A_{2}=\eta c_{q} / D_{q}, c_{q}=\hbar v\left(q_{x}^{2}+k_{y}^{2}\right)^{1 / 2}$, and $D_{q}=\left[c_{q}^{2}+\right.$ $\left.\left[-\Delta-\eta s_{z}\left(\lambda_{c}-\lambda_{v}\right)+t E_{s_{z}}^{\eta}\left(q_{x}, k_{y}\right)\right]^{2}\right]^{1 / 2}$. Also, $B_{2}=b_{q} / D_{q}$ and $b_{q}=-\Delta-\eta s_{z}\left(\lambda_{c}-\lambda_{v}\right)+t E_{s_{z}}^{\eta}\left(q_{x}, k_{y}\right)$. Further, $\theta$ and $\varphi$ are the angles of incidence and reflection given by $\theta=$ $\tan ^{-1}\left(k_{y} / k_{x}\right), k_{x}=k_{F} \cos \theta$, and $k_{y}=k_{F} \sin \theta$, with $k_{F}$ the Fermi momentum, $q_{x}=\left[k_{F}^{\prime 2}-k_{y}^{2}\right]^{1 / 2}$, and $\varphi=\tan ^{-1}\left(k_{y} / q_{x}\right)$. In general, $k_{F}^{\prime}$ includes the changes in potential or/and Zeeman field in region II. As usual, the translation invariance along the $y$ axis implies that the wave vector $k_{y}$ is conserved.

The coefficients $r_{\eta s_{z}}, P, Q$, and $t_{\eta s_{z}}$ are determined by the continuity of the wave functions, i.e., by

$$
\Psi_{I}(0-, y)=\Psi_{I I}(0+, y), \Psi_{I I}(d-, y)=\Psi_{I I I}(d+, y) .
$$

The resulting transmission $T_{\eta s_{z}}=\left|t_{\eta s_{z}}\right|^{2}$ reads

$$
T_{\eta s_{z}}=1 /\left[1+\sin ^{2}\left(q_{x} d\right)\left(F^{2}(\gamma, \theta, \varphi)-1\right],\right.
$$

where $\gamma=k_{F} b_{q} /\left(k_{F}^{\prime} b_{k}\right)$ and $F(\gamma, \theta, \varphi)=\left(\gamma+\gamma^{-1}-\right.$ $2 \sin \theta \sin \varphi) /(2 \cos \theta \cos \varphi)$.

Now we are ready to evaluate the conductance [27] due to a particular spin and valley; it is given by

$$
G_{\eta s_{z}}=G_{0} \int_{-\pi / 2}^{\pi / 2} T_{\eta s_{z}}(\theta) \cos \theta d \theta=G_{0} g_{c \eta s_{z}},
$$

where $G_{0}=e^{2} k_{F} W /(2 \pi h)$ and $W$ is the width along the $y$ direction. Furthermore, the spin $P_{s}$ and valley $P_{v}$ polarizations are defined as

$$
P_{s}=\frac{g_{c \uparrow}-g_{c \downarrow}}{g_{c \uparrow}+g_{c \downarrow}} \quad \text { and } \quad P_{v}=\frac{g_{c K}-g_{c K^{\prime}}}{g_{c K}+g_{c K^{\prime}}} .
$$

Below we will present various results for the conductance and polarizations. In the calculations, we use the Fermi energy $E_{F}$ for which both spin states and valleys in regions I and III are occupied.

\section{NUMERICAL RESULTS AND DISCUSSION}

In Fig. 3, we show the total conductance $g_{c}$ (solid black curve) versus the barrier width $d$ and its particular spin and valley contributions. The red dashed curve is for the $K$ valley,

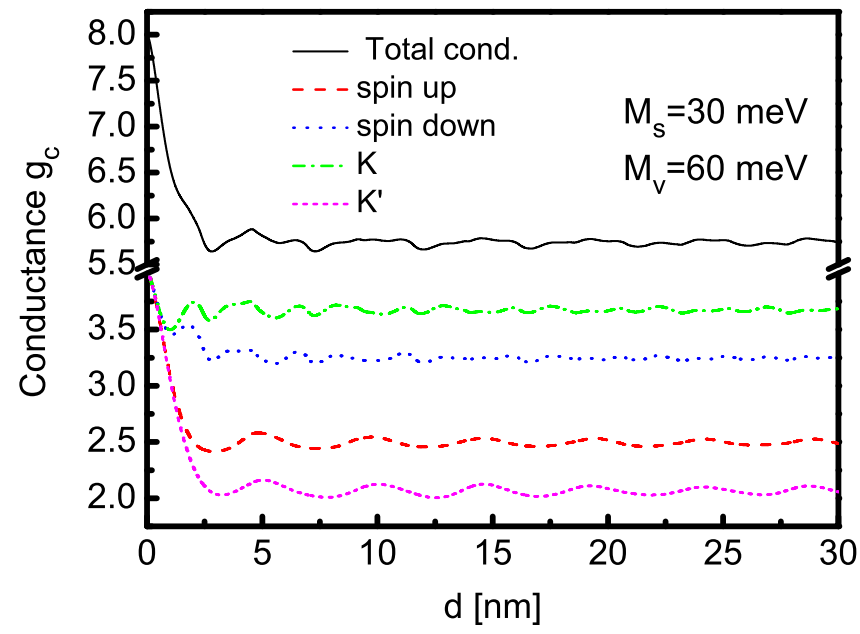

FIG. 3. Total (normalized) conductance (solid black curve) vs barrier width $d$ with its spin and valley components as indicated. The parameters are $E_{F}=0.95 \mathrm{eV}, U=0 \mathrm{meV}, M_{s}=30 \mathrm{meV}$, and $M_{v}=60 \mathrm{meV}$.

the green dotted one for the $K^{\prime}$ valley. The spin-up contribution is shown by the blue dash-dotted curve and the spin-down one by the magenta short-dashed curve. The Fermi level is $E_{F}=0.95 \mathrm{eV}$, while $M_{s}=30 \mathrm{meV}$ and $M_{v}=60 \mathrm{meV}$. While the particular contributions show nice and regular oscillations versus $d$, the total conductance $g_{c}$ is rather irregular. In all cases, $g_{c}$ has large values for very small barrier widths $d$. If we interchange the values of $M_{s}$ and $M_{v}$, we obtain the same total conductance but with the $K$ and $K^{\prime}$ contributions as well as the spin-up and spin-down ones interchanged.

Next we present results in Fig. 4 for the spin $P_{S}$ (red solid curve) and valley $P_{v}$ (blue dashed curve) polarizations versus barrier width $d$ for $M_{s}=30 \mathrm{meV}$ and $M_{v}=60 \mathrm{meV}$ (left panels) and $M_{s}=60 \mathrm{meV}$ and $M_{v}=30 \mathrm{meV}$ (right panels). Also two values of the potential barrier are considered: $U=0 \mathrm{meV}$ (upper panels) and $U=45 \mathrm{meV}$ (lower panels). Both types of polarization start from zero, i.e., there is no
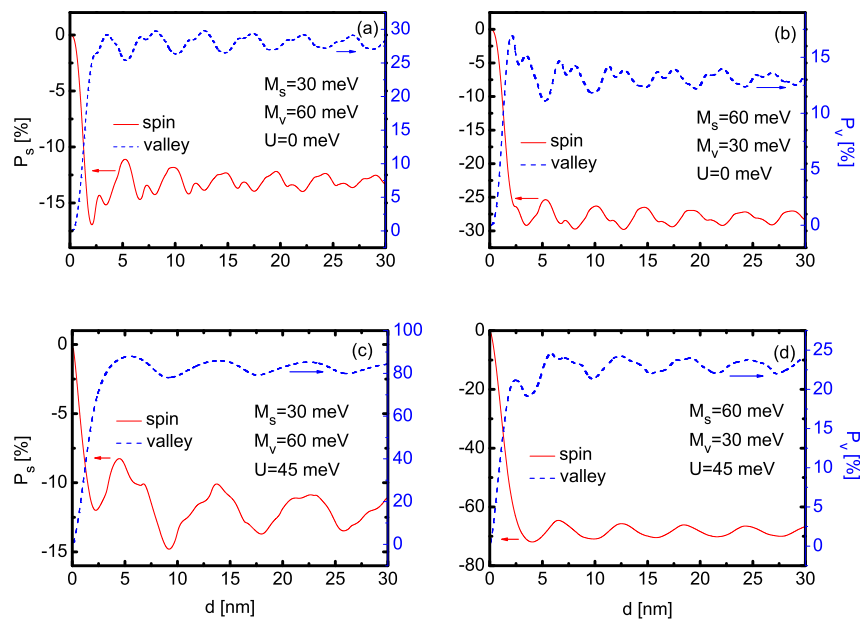

FIG. 4. Spin (red curve) and valley (blue) polarization as a function of the barrier width $d$, for $M_{s}=30 \mathrm{meV}, M_{v}=60 \mathrm{meV}$ (left) and $M_{s}=60 \mathrm{meV}, M_{v}=30 \mathrm{meV}$ (right). The upper panels are for $U=0$, the lower ones for $U=45 \mathrm{meV}$, and all for $E_{F}=0.95 \mathrm{eV}$. 

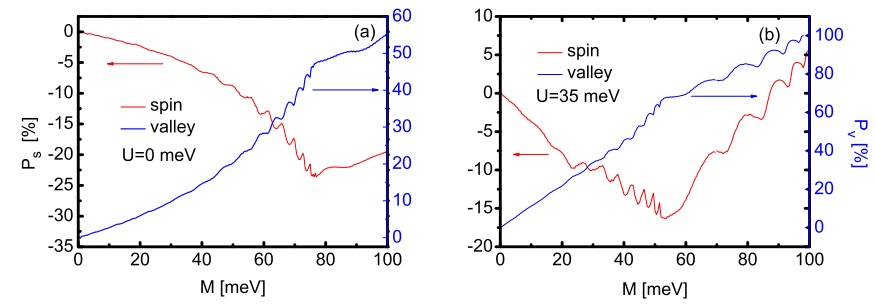

FIG. 5. Polarizations, as indicated, vs the Zeeman field $2 M_{s}=$ $M_{v}=\mathrm{M}$, for (a) $U=0 \mathrm{meV}$, (b) $U=35 \mathrm{meV}$, and $E_{F}=0.95 \mathrm{meV}$, $d=30 \mathrm{~nm}$ in all panels.

polarization for vanishing $d$ as expected. For small values of $d$, both polarizations increase and attain some values around, which they oscillate. In both cases, the oscillations are, in principle, the result of two oscillating terms that depend on different propagating wave vectors for two different spin/valley indices in region II. Furthermore, it can be seen that with an applied voltage one can increase $P_{v}$ above $80 \%$ for this value of the Zeeman field $M$.

Next, we vary the magnetization $2 M_{s}=M_{v}=M$ and inspect polarizations (Fig. 5) at fixed barrier width $d=30 \mathrm{~nm}$. The left panel is for zero potential $U=0 \mathrm{meV}$ and the right one for $U=35 \mathrm{meV}$. For $U=0 \mathrm{meV}$, the polarizations $P_{s}$ and $P_{v}$ rise in absolute value until $M=M_{\mathrm{cr}}$, and then $P_{s}$ slowly declines in absolute value. Notice though that $P_{v}$ attains a high value, approximately $55 \%$ for $M=100 \mathrm{meV}$. However, when a potential difference $(U=35 \mathrm{meV})$ is present the values of $P_{v}$ becomes larger and even reaching $100 \%$ for $\mathrm{M}$ close to $100 \mathrm{meV}$. In contrast, $P_{s}$ exhibits a nonmonotonic behavior with a maximum at around $M=50 \mathrm{meV}$. At this point, we emphasize the difference with the case in which $\mathrm{WSe}_{2}$ is replaced by $\mathrm{MoS}_{2}$ considered in our previous work [29]. In the case of $\mathrm{MoS}_{2}, P_{v}$ could not rise above $10 \%$ even for $M=100 \mathrm{meV}$. This is in contrast to the present case in which $P_{v}$ can reach $25 \%$ even for moderate Zeeman fields $(\mathrm{M}=60 \mathrm{meV})$. There are two reasons for this difference: (i) the spin-orbit coupling in $\mathrm{MoS}_{2}$ is only $37.5 \mathrm{meV}$, which is at least three times less than in $\mathrm{WSe}_{2}$, and (ii) there is no Zeeman field for the valley degree of freedom.

We proceed with Fig. 6 in which we show the conductance $g_{c}$ versus the parameter $k_{F} d$, where $k_{F}$ is the Fermi wave

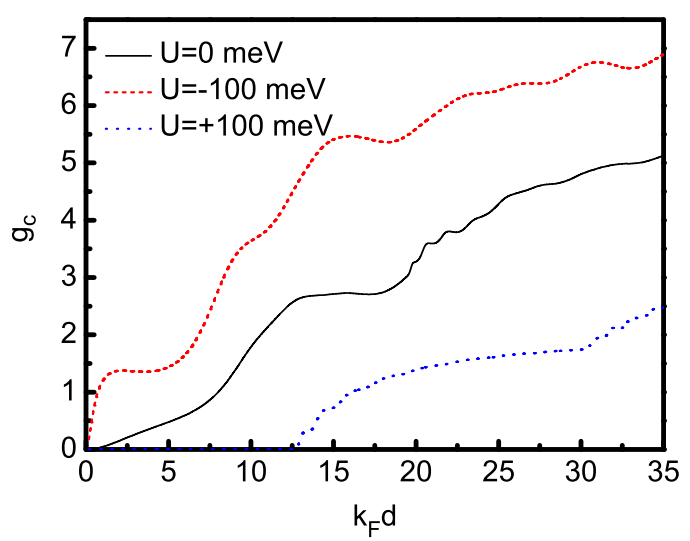

FIG. 6. Total conductance vs $k_{F} d$ for three values of the barrier potential $U$ as indicated. The parameters are $E_{F}=0.95 \mathrm{eV}, M_{s}=30$ $\mathrm{meV}$, and $M_{v}=60 \mathrm{meV}$.

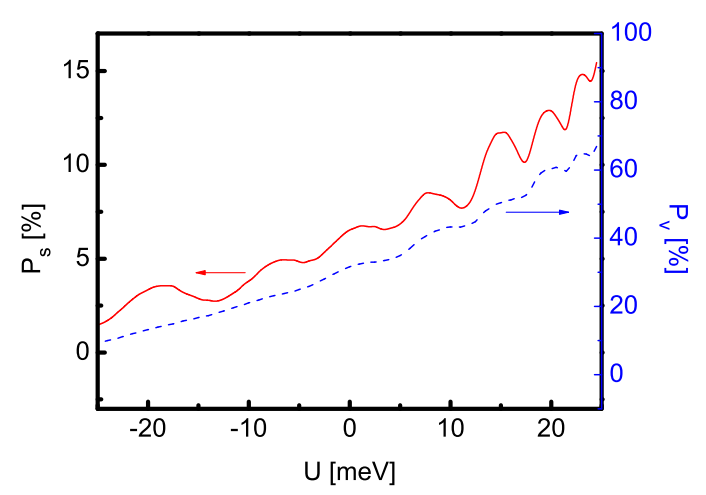

FIG. 7. Spin $P_{s}$ and valley $P_{v}$ polarizations vs barrier potential $U$ for $E_{F}=0.95 \mathrm{eV}, d=30 \mathrm{~nm}, M_{s}=0 \mathrm{meV}$, and $M_{v}=60 \mathrm{meV}$.

vector, for three values of the potential barrier $U$ : the red dashed, solid black, and blue dotted curves are for $U=-100$ $\mathrm{meV}, U=0 \mathrm{meV}$, and $U=+100 \mathrm{meV}$, respectively. The other parameters are $E_{F}=0.95 \mathrm{eV}, M_{s}=30 \mathrm{meV}$, and $M_{v}=60 \mathrm{meV}$. A positive potential barrier impedes the current so that the conductance is smaller for $U=+100$ $\mathrm{meV}$, while for $U=-100 \mathrm{meV}$ is larger than that of the usual case $U=0 \mathrm{meV}$ when there is no potential barrier. For shorter $k_{F}\left(k_{F} d<12\right)$, the effect of a positive potential $U$ is even completely suppressed. The other two cases show mild oscillations versus $k_{F} d$.

Further, we investigate the influence of applying a voltage $U$ in region II while keeping other parameters fixed. In Fig. 7, we show $P_{s}$ and $P_{v}$ as functions of the applied voltage $U$ for $E_{F}=0.95 \mathrm{eV}, d=30 \mathrm{~nm}, M_{s}=0 \mathrm{meV}$ and $M_{v}=60 \mathrm{meV}$. Both polarizations rise with increasing voltage $U$, while the values of $P_{v}$ are larger than those of $P_{s}$ because we assumed $M_{v} \gg M_{s}$.

In order to investigate the combined influence of the Zeeman fields and applied voltage on both polarizations we show contour plots of $P_{s}$ in Fig. 8(a) and of $P_{v}$ in Fig. 8(b) for $M_{s}=M_{v}$. We see that $P_{v}$ becomes perfect for $M \geqslant 15$ meV and large $U \geqslant 90 \mathrm{meV}$ (magenta region), while $P_{s}$ attains mostly large negative values for $M \geqslant 15 \mathrm{meV}$ and $U \geqslant 90 \mathrm{meV}$ (dark blue region).

We now turn our attention to a double barrier case, that is when two magnetic layers are separated by a normal layer (all made of $\mathrm{WSe}_{2}$ ) and have different magnetization and potential
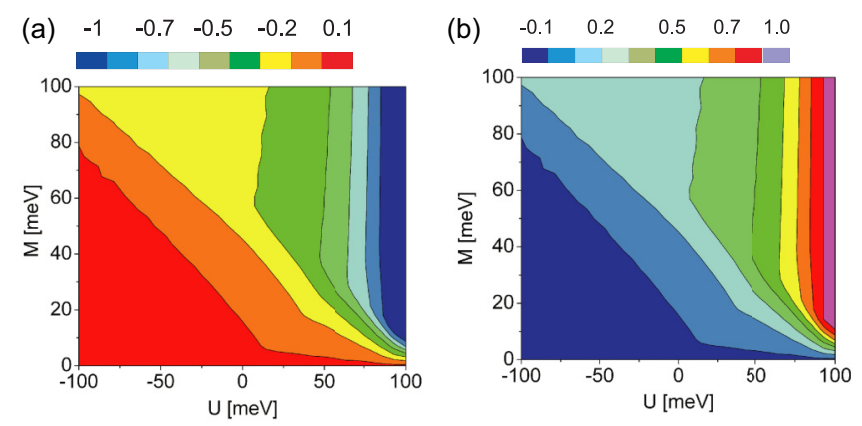

FIG. 8. $(M, U)$ contour plots of (a) spin polarization; (b) valley polarization. Both panels are for $E_{F}=0.95 \mathrm{eV}$ and barrier width $d=30 \mathrm{~nm}\left(M_{s}=M_{v}\right)$. 

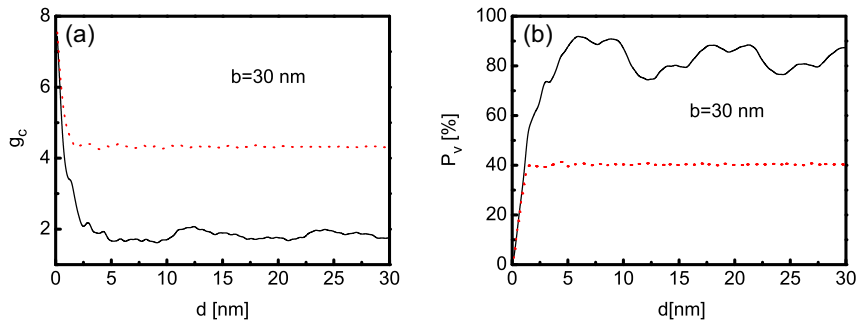

FIG. 9. (a) Total conductance $g_{c}$ and (b) valley polarization $P_{v}$ vs barrier width $d$ in a double barrier setup with $U=40 \mathrm{meV}$, barrier separation $b=30 \mathrm{~nm}$, and $M_{v}=M_{s}=60 \mathrm{meV}$. The solid black curve and the red dotted one are for $E_{F}=0.95$ and $1.05 \mathrm{eV}$, respectively.

energies ( $2 M, 2 U$ in the second barrier and $M_{s}=M_{v}=M$ in the first). In Fig. 9(a), we show the total conductance $g_{c}$ when the barrier width is varied for $U=40 \mathrm{meV}$. The solid black curve is for $E_{F}=0.95 \mathrm{eV}$ and the red dotted one for $E_{F}=$ $1.05 \mathrm{eV}$. The values and overall $d$ dependence are similar to the single-barrier case (cf. Fig. 3), although the local maxima occur at lower values (at around 2). As seen, upon increasing $E_{F}$, the conductance rises since there are more available states for tunneling but, as Fig. 9(b) shows, $P_{v}$ decreases since the occupation of the other valley's subbands increases with $E_{F}$.

Next, we plot the spin (red curve) and valley (blue curve) polarization versus the Zeeman field $M_{v}=M_{s}=M$ in Fig. 10 . The left panel is for $U=20 \mathrm{meV}$ and the right one for $U=40 \mathrm{meV}$. The solid and dotted curves are for $E_{F}=0.95$ and $1.05 \mathrm{eV}$, respectively. Both polarizations exhibit less monotonic behavior and, similar to Fig. 9, they decrease with $E_{F}$. As in Fig. 5, these results show that with an appropriate applied voltage one can increase the valley polarization close to $100 \%$. Further, increasing $E_{F}$ leads to a decrease of $P_{v}$ and $P_{s}$ due to an increased occupation of the corresponding opposite subbands. In general, this decrease of $P_{v}$ and $P_{s}$ with increasing $E_{F}$ occurs for single junctions as well.

Further, in Fig. 11, we show contour plots of $P_{s}$ and $P_{v}$ for the double barrier case when both the Zeeman field $M$ and the potential difference $U$ are varied. Comparing with the single barrier case one can conclude that high values of $P_{s}$ and $P_{v}$ (in absolute sense) are obtainable in a wider region of the $(M, U)$ plane. One can see that perfect $P_{v}$ is achievable for $\mathrm{M} \geqslant 5 \mathrm{meV}$ and $U \geqslant 50 \mathrm{meV}$ (red region).
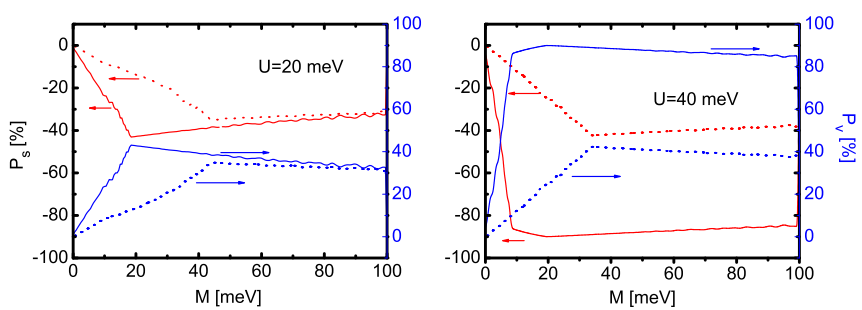

FIG. 10. Polarizations, as indicated, as a function of the Zeeman field $M_{v}=M_{s}=M$. The left panel is for $U=20$, while the right panel is for $U=40 \mathrm{meV}$. The solid and dotted curves are for $E_{F}=0.95$ and $1.05 \mathrm{eV}$, respectively.
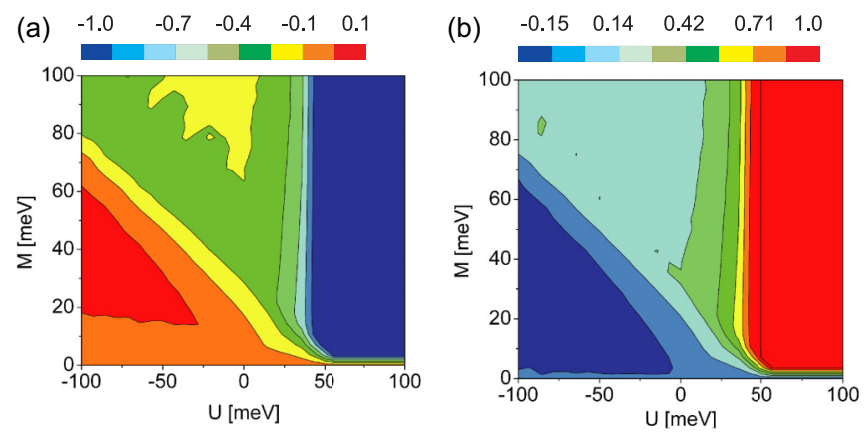

FIG. 11. $(M, U)$ contour plots for the double barrier case: (a) spin polarization, (b) valley polarization. Both panels are for $E_{F}=$ $0.95 \mathrm{eV}$, barriers' width $d=30 \mathrm{~nm}$, and barrier separation $b=30 \mathrm{~nm}$.

Given all results presented thus far one may wonder whether the frequently used values $M_{s}=30 \mathrm{meV}$ and $M_{v}=60 \mathrm{meV}$ are too high and whether one needs to apply a strong magnetic field in order to obtain such splittings. This need not be the case because firstly, as shown in the $(M, U)$ contour plots, one can have $1 \geqslant P_{s}>0.7$ and $1 \geqslant P_{v}>0.7$ for $M \geqslant 10 \mathrm{meV}$ and $U \geqslant 75 \mathrm{meV}$ in Fig. 8, whereas in Fig. 11 this occurs for $M \geqslant 5 \mathrm{meV}$ and $U \geqslant 45 \mathrm{meV}$. Secondly, first-principle calculations $[10,30]$ show that one can obtain very large spin and valley splittings in $\mathrm{MoTe}_{2} / \mathrm{EuO}$ without applying a magnetic field. A valley splitting of $300 \mathrm{meV}$ is reported in Ref. [28] for $\mathrm{MoTe}_{2} / \mathrm{EuO}$ and an effective exchange field of $160 \mathrm{meV}$ for $\mathrm{MoTe}_{2} / \mathrm{EuO}(111)$ in Ref. [10]. The similarity of these systems with, e.g., $\mathrm{WSe}_{2} / \mathrm{EuO}$, imply that similar splittings, though probably weaker than in $\mathrm{MoTe}_{2} / \mathrm{EuO}$, can occur, e.g, in $\mathrm{WSe}_{2} / \mathrm{EuO}$ or $\mathrm{WSe}_{2} / \mathrm{EuO}(111)$. Recently, the applicability and usefulness of the magnetic proximity effect, using TMDCs on magnetic semiconductor substrates, has been reported in Ref. [31].

Finally, a comment is in order on the feasibility of the tunneling barrier structures we considered. From exciton studies in high-quality monolayer $\mathrm{MoS}_{2}$ samples a mobility $\mu=311 \mathrm{~cm}^{2} \mathrm{~V}^{-1} \mathrm{~s}^{-1}$ and a phase coherence length $L_{\phi}$ of 50 to $100 \mathrm{~nm}$ have been reported in Ref. [32]. Also, from similar studies in monolayer $\mathrm{WSe}_{2}$ and with a scattering time of $200 \mathrm{fs}$, see Ref. [33], we estimate a mobility $\mu=600 \mathrm{~cm}^{2} \mathrm{~V}^{-1} \mathrm{~s}^{-1}$ and a phase coherence length $L_{\phi} \approx 50 \mathrm{~nm}$. We assume that these results or estimates for $\mu$ and $L_{\phi}$ remain valid for ballistic transport and junction widths $1 \mathrm{~nm}$ to $30-\mathrm{nm}$ long that we considered. Given that Refs. $[24,25]$ are initial experiments and further experimental progress is expected, we think that such junctions will be soon realized experimentally and the magnetic [31] control of the valley degree of freedom will be tested.

\section{SUMMARY}

We studied magnetic- and electric-field-controlled spinand valley-polarized transport through biased, single or double tunnel junctions on monolayer $\mathrm{WSe}_{2}$. The degree of $\operatorname{spin}\left(P_{s}\right)$ and valley $\left(P_{v}\right)$ polarization depends on the values of $M_{s}, M_{v}$, and $U$. We showed that fully valley- and spin-polarized currents can be obtained simultaneously in this device, cf. Figs. 9-11. We also showed spin- and valley-resolved 
conductances and clarified how the valley Zeeman field leads to the polarization $P_{v}$. Physically, in $\mathrm{WSe}_{2}$ junctions, the Zeeman field opens different spin-dependent band gaps at the $K$ and $K^{\prime}$ valleys, which in turn induce spin- and valleypolarized transport. Interchanging the values of $M_{s}$ and $M_{v}$ leads to the interchange of $P_{s}$ and $P_{v}$ but the total conductance remains the same. All these results confirm the magnetic and electric control of the polarizations and contribute significantly to the fundamental investigations of the electronic properties of $2 \mathrm{D} \mathrm{WSe} e_{2}$. As such, they may be quite relevant to the design of spintronic and valleytronic devices.

\section{ACKNOWLEDGMENTS}

This work was supported by the University of Hafr Al Batin (MT), the Ministry of Education, Science, and Technological development of Serbia within the Project No. TR 32008 (PMK), and the Canadian NSERC Grant No. OGP0121756 (PV).
[1] X. Xu, W. Yao, D. Xiao, and T. F. Heinz, Nat. Phys. 10, 343 (2014).

[2] K. S. Novoselov, A. K. Geim, S. Morozov, D. Jiang, Y. Zhang, S. Dubonos, I. Grigorieva, and A. A. Firsov, Science 306, 666 (2004).

[3] A. H. Castro Neto, F. Guinea, N. M. R. Peres, K. S. Novoselov, and A. K. Geim, Rev. Mod. Phys. 81, 109 (2009).

[4] L. Liao, Y.-C. Lin, M. Bao, R. Cheng, J. Bai, Y. Liu, Y. Qu, K. L. Wang, Y. Huang, and X. Duan, Nature 467, 305 (2010); F. Schwierz, Nat. Nanotechnol. 5, 487 (2010).

[5] A. K. Geim and I. V. Grigorieva, Nature 499, 419 (2013).

[6] H. Fang, S. Chuang, T. C. Chang, K. Takei, T. Takahashi, and A. Javey, Nano Lett. 12, 3788 (2012); H. Wang, L. Yu, Y.-H. Lee, Y. Shi, A. Hsu, M. L. Chin, L.-J. Li, M. Dubey, J. Kong, and T. Palacios, ibid. 12, 4674 (2012); M. S. Fuhrer and J. Hone, Nat. Nanotechnol. 8, 146 (2013).

[7] D. Xiao, G.-B. Liu, W. Feng, X. Xu, and W. Yao, Phys. Rev. Lett. 108, 196802 (2012).

[8] H.-Z. Lu, W. Yao, D. Xiao, and S.-Q. Shen, Phys. Rev. Lett. 110, 016806 (2013).

[9] X. Li, F. Zhang, and Q. Niu, Phys. Rev. Lett. 110, 066803 (2013).

[10] Q. Zhang, S. A. Yang, W. Mi, Y. Cheng, and U. Schwingenschlögl, Adv. Mater. 28, 959 (2016).

[11] A. Splendiani, L. Sun, Y. Zhang, T. Li, J. Kim, C.-Y. Chim, G. Galli, and F. Wang, Nano Lett. 10, 1271 (2010).

[12] J. K. Ellis, M. J. Lucero, and G. E. Scuseria, Appl. Phys. Lett. 99, 261908 (2011).

[13] H. S. Lee, S.-W. Min, Y.-G. Chang, M. K. Park, T. Nam, H. Kim, J. H. Kim, S. Ryu, and S. Im, Nano Lett. 12, 3695 (2012).

[14] E. Cappelluti, R. Roldán, J. A. Silva-Guillén, P. Ordejón, and F. Guinea, Phys. Rev. B 88, 075409 (2013).

[15] B. Radisavljevic, A. Radenovic, J. Brivio, V. Giacometti, and A. Kis, Nat. Nanotechnol. 6, 147 (2011).

[16] Q. H. Wang, K. Kalantar-Zadeh, A. Kis, J. N. Coleman, and M. S. Strano, Nat. Nanotechnol. 7, 699 (2012).
[17] H. Liu, A. T. Neal, and P. D. Ye, ACS Nano 6, 8563 (2012).

[18] Y. Yoon, K. Ganapathi, and S. Salahudin, Nano Lett. 11, 3768 (2011).

[19] H. Li, J. Shao, D. Yao, and G. Yang, ACS Appl. Mat. Interfaces 6, 1759 (2014).

[20] T. Yokoyama, Phys. Rev. B 87, 241409(R) (2013).

[21] C. Vargiamidis and P. Vasilopoulos, J. Appl. Phys. 117, 094305 (2015); Appl. Phys. Lett. 105, 223105 (2014).

[22] N. Missault, P. Vasilopoulos, V. Vargiamidis, F. M. Peeters, and B. Van Duppen, Phys. Rev. B 92, 195423 (2015).

[23] A. M. Jones, H. Yu, J. S. Ross, P. Klement, N. J. Ghimire, J. Yan, D. G. Mandrus, W. Yao, and X. Xu, Nat. Phys. 10, 130 (2014).

[24] G. Aivazian, Z. Gong, A. M. Jones, R.-L. Chu, J. Yan, D. G. Mandrus, C. Zhang, D. Cobden, W. Yao, and X. Xu, Nat. Phys. 11, 148 (2015).

[25] A. Srivastava, M. Sidler, A. V. Allain, D. S. Lembke, A. Kis, and A. Imamoğlu, Nat. Phys. 11, 141 (2015).

[26] H. C. P. Movva, A. Rai, S. Kang, K. Kim, B. Fallahazad, T. Taniguchi, K. Watanabe, E. Tutuc, and S. K. Banerjee, ACS Nano 9, 10402 (2015).

[27] F. Zhai and K. Chang, Phys. Rev. B 85, 155415 (2012).

[28] C. Zhao, T. Norden, P. Zhang, P. Zhao, Y. Cheng, F. Sun, J. P. Parry, P. Taheri, J. Wang, Y. Yang, T. Scrace, K. Kang, S. Yang, G.-x. Miao, R. Sabirianov, G. Kioseoglou, W. Huang, A. Petrou, and H. Zeng, Nat. Nanotechnol. (2017), doi:10.1038/nnano.2017.68.

[29] P. M. Krstajić, M. Tahir, and P. Vasilopoulos, Physica E (Amsterdam) 75, 317 (2016).

[30] J. Qi, X. Li, Q. Niu, and J. Feng, Phys. Rev. B 92, 121403(R) (2015).

[31] Y. Ye, J. Xiao, H. Wang, Z. Ye, H. Zhu, M. Zhao, Y. Wang, J. Zhao, X. Yin, and X. Zhang, Nat. Nanotechnol. 11, 598 (2016).

[32] A. T. Neal, H. Liu, J. Gu, and P. D. Ye, ACS Nano 7, 7077 (2013).

[33] K. Hao, G. Moody, F. Wu, C. K. Dass, L. Xu, C.-H. Chen, L. Sun, M.-Y. Li, L.-J. Li, A. H. MacDonald, and X. Li, Nat. Phys. 12, 677 (2016). 